\title{
Méthode simplifiée pour calculer la récupération optimum d'énergie sur un pasteurisateur de lait à plaques
}

\author{
par \\ G. CORRIEU*, M. LALANDE* et C. ROUSSEL**
}

\section{INTRODUCTION}

Les échangeurs de chaleur à plaques constituent la majeure partie des pasteurisateurs utilisés dans l'industrie laitière. Nous avons, dans un passé récent, décrit les performances de certains de ces matériels [8] et analysé les principaux avantages expliquant leur succès [2]. Les pasteurisateurs industriels se caractérisent par une zone importante de récupération d'énergie au sein de laquelle le lait chaud réchauffe le lait froid assurant une appréciable économie d'énergie. La figure 1 représente schématiquement cette propriété.

Ces dernières années l'augmentation rapide des coûts de l'énergie sous toutes ses formes a rendu rentable la mise en œuvre de zones de récupération de plus en plus grandes. Warshney et al. [12] illustrent ce propos d'un exemple significatif dans le cas de la pasteurisation de la crème. Derkenne [3] rapporte que dans le cas de la pasteurisation du lait, la récupération de plus de 85 p. 100 de l'énergie nécessaire au chauffage du lait (entre sa température d'entrée et sa température de pasteurisation) est quasi générale. En réalité, les installations de grande taille fonctionnent déjà avec des pourcentages de récupération supérieurs à 90 p. 100 .

Les possibilités de modification des échangeurs à plaques, qui permettent de faire évoluer dans le temps les programmes thermiques mis en œuvre, ont favorisé cette évolution puisqu'il suffit d'augmenter la surface d'échange de la zone de récupération dans les proportions adéquates c'est-à-dire d'ajouter le nombre de plaques

* I.N.R.A., Laboratoire de Génie Industriel, 369, rue J.-Guesde 59650 Villeneuve-d'Ascq.

** Société Vicarb, 24, avenue M.-Cachin - 38400 Saint-Martin-d'Hères. 
voulu. Une difficulté pratique peut apparaître sur les pasteurisateurs existants lorsque le bâti de l'échangeur se trouve garni complètement et ne permet plus d'augmenter la surface de récupération de chaleur.

Mais le vrai problème est de définir avec exactitude le pourcentage optimum d'énergie à récupérer. En effet, il apparaît que la surface d'échange nécessaire à des récupérations d'énergies supérieures à 90 p. 100 augmente très vite [2]. Ce phénomène trouve son explication partielle dans la réduction des différences de température entre les deux courants de lait au fur et à mesure que le pourcentage de récupération augmente. Comme l'investissement est proportionnel à la surface installée, à partir d'un certain seuil, son coût ne sera plus compensé par l'économie d'énergie. Il est logique de penser que la somme du coût de l'énergie et du coût de l'investissement va présenter une valeur minimum pour un pourcentage de récupération qui sera défini comme l'optimum de récupération à mettre en œuvre.

Ainsi les utilisateurs de pasteurisateurs se trouvent confrontés à un véritable problème d'optimisation.

Parmi l'abondante littérature scientifique consacrée aux travaux d'optimisation de procédés, rien ne se rapporte au cas de la pasteurisation d'un produit alimentaire. Par contre, plusieurs chercheurs se sont intéressés à l'optimisation des échangeurs de chaleur de toute nature. Certains, sans réellement intégrer des critères économiques dans leur raisonnement, ont proposé des vitesses idéales de circulation des fluides [5] ou ont défini différents paramètres à optimiser tels le rapport coefficient d'échange-puissance de pompage [11] ou l'efficacité thermique [1].

Plus souvent les critères économiques sont pris en compte afin de définir les géométries et les conditions de fonctionnement optimales $[11,10,7]$. Des moyens informatiques importants sont alors indispensables car la difficulté majeure du problème résulte du très grand nombre de paramètres à considérer.

Ainsi, en prenant l'exemple d'une unité industrielle de pasteurisation et en admettant l'utilisation d'un échangeur à plaques (ce qui élimine nombre de paramètres géométriques), le coût à minimiser doit considérer les paramètres suivants :

- débit de lait ;

- programme thermique (en particulier température de pasteurisation) ;

- pourcentage d'énergie récupérée ;

- durée journalière de travail ;

- degré d'automatisation de l'installation ;

— durée d'amortissement de l'investissement ; 
- mode de rinçage et de nettoyage ;

- sources d'énergie utilisées.

En pratique, une fonction coût intégrant tous ces paramètres devra être établie pour chaque type de dépenses (investissement, entretien, main-d'œuvre, énergie, eau, réactifs chimiques, fournitures). N'insistons pas sur la complexité du problème et la difficulté des solutions.

En conséquence, dans certains cas, une simplification par réduction du nombre de paramètres sera effectuée. Cette opération nécessite un soin particulier afin d'éviter des erreurs graves.

La méthode que nous présentons pour calculer le pourcentage de récupération d'énergie sur un pasteurisateur se veut un exemple de ce type de simplification.

\section{PRESENTATION DE LA METHODE}

\section{Hypothèses simplificatrices}

Deux des éléments qui composent une unité industrielle de pasteurisation de lait peuvent être optimisés de façon indépendante. L'un est la conduite des opérations de nettoyage, l'autre la gestion de l'énergie.

Pour justifier cette démarche, il ne faut pas perdre de vue l'intérêt économique de l'utilisateur qui cherche à traiter le lait au moindre coût afin de dégager une plus-value maximum.

Si nous considérons que l'installation est rentable en soi, l'optimisation du nettoyage et des dépenses énergétiques ne peut qu'entraîner une rentabilité accrue. Ce raisonnement permet de traiter séparément le problème de l'optimisation de la récupération d'énergie dépensée et le coût de l'investissement spécifique nécessaire à la récupération de l'énergie.

Après avoir admis de traiter séparément l'aspect énergétique de la pasteurisation, nous proposons deux hypothèses simplificatrices :

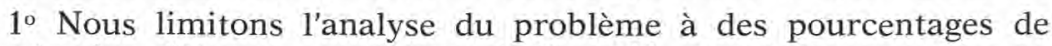
récupération d'énergie supérieurs à 80 p. 100, c'est-à-dire que l'investissement correspondant à une récupération de 80 p. 100 de l'énergie est pris comme référence et que nous ne raisonnons que sur le coût de l'investissement supplémentaire assurant des récupérations supérieures.

Sur le plan économique, cela signifie que le bénéfice retiré du procédé doit permettre d'amortir le coût d'investissement du pasteurisateur dans sa configuration récupérant 80 p. 100 de l'énergie. $\mathrm{Au}$ delà de ce pourcentage, les coûts de l'énergie et de l'investissement supplémentaires conduisent à la définition d'un pourcentage de 
récupération optimum qu'il serait bon de mettre en œuvre. Cet optimum est la valeur du pourcentage de récupération rendant minimum la somme des deux coûts.

$2^{\circ}$ Quel que soit le pourcentage de récupération étudié, nous avons calculé les surfaces d'échange des différentes zones de l'échangeur afin de maintenir la perte de charge du circuit à 2 bars $\pm 0,1$. Cette procédure permet de négliger les coûts de pompage puisque la même pompe est utilisée quelle que soit l'importance de la zone de récupération. Dans l'hypothèse contraire, lorsque la surface de cette zone augmente, le maintien de la vitesse de circulation assurant un coefficient de transfert de chaleur constant se traduirait par un accroissement considérable de la perte de charge. Dans l'industrie de telles pertes de charge ne sont guère acceptables. En conséquence, la configuration de l'échangeur est aménagée dans le sens d'une diminution de la vitesse de circulation et du coefficient de transfert ; cela se traduit par un accroissement encore plus important de la surface d'échange.

\section{Définition des fonctions coûts}

Ces hypothèses étant admises, il ne reste plus qu'à établir les deux fonctions traduisant respectivement le coût de l'énergie dépensée et le coût de l'investissement supplémentaire en fonction du pourcentage d'énergie récupérée.

\section{2a COÛT DE L'ÉNERGIE DÉPENSÉE}

Soit $\mathrm{E}_{\mathrm{r}}$, l'énergie nécessaire pour amener le lait de sa température d'entrée à sa température de pasteurisation :

$\mathrm{E}_{\mathrm{T}}=\rho . \mathrm{D} . \mathrm{Cp} . \Delta \mathrm{T}$ (voir la table des symboles et unités)

Si le pourcentage de récupération $(\% \mathrm{R})$ est défini comme la fraction de $\mathrm{E}_{\mathrm{r}}$ récupérée par réchauffage du lait froid par le lait chaud, l'énergie dépensée pour le chauffage $\left(\mathrm{E}_{\mathrm{cн}}\right)$ sera :

$$
\mathrm{E}_{\mathrm{CH}}=\frac{\mathrm{E}_{\mathrm{r}}(100-\% \mathrm{R})}{100}
$$

Si comme l'indique la figure 1, le lait est ensuite réfrigéré, il est possible de définir en fonction de la température de sortie du lait, une énergie de réfrigération ( $\mathrm{E}_{\mathrm{kF}}$ ) qui s'exprime par une équation de la même forme que celle exprimant $\mathrm{E}_{\mathrm{r}}$. Dans le cas particulier où le lait est refroidi jusqu'à atteindre sa température d'entrée, les énergies de réfrigération et de chauffage sont égales ; d'où l'expression de l'énergie totale dépensée par le procédé $\left(\mathrm{E}_{\mathrm{n}}\right)$ :

$$
\mathrm{E}_{\mathrm{D}}=\mathrm{E}_{\mathrm{CH}}+\mathrm{E}_{\mathrm{RE}}=\frac{2 \mathrm{E}_{\mathrm{T}}(100-\% \mathrm{R})}{100}
$$

C'est cette équation que nous utiliserons par la suite. Cela revient à dire que l'énergie mise en œuvre pour augmenter la température du 


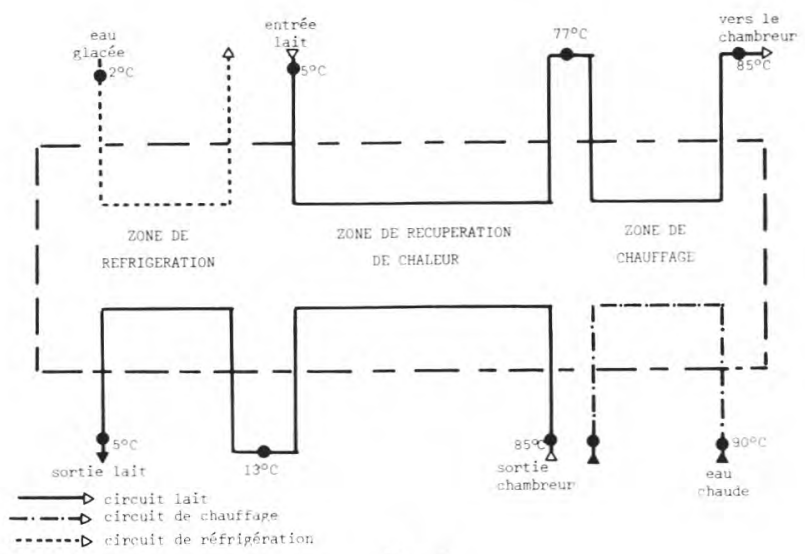

fig. 1

Schéma de fonctionnement d'un pasteurisateur à plaques et programme thermique associé.

lait est égale à celle mise en œuvre pour diminuer sa température. Les pourcentages de récupération de chaleur, en chauffage et en réfrigération, sont égaux.

Remarquons aussi que ce raisonnement néglige les pertes thermiques propres au procédé. Néanmoins, un coefficient correctif pourrait facilement être introduit.

En considérant les prix de la thermie $\left(\mathrm{P}_{\mathrm{t}}\right)$ et du kilowatt-heure $\left(\mathrm{P}_{\mathrm{e}}\right)$, le rendement de la machine frigorifique $(\varepsilon)$ et le nombre annuel d'heures de fonctionnement $(\mathrm{N})$, nous exprimons les coûts annuels du chauffage et de la réfrigération selon :

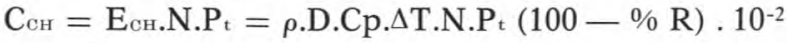

$$
\begin{aligned}
& \mathrm{C}_{\mathrm{RE}}=\frac{\mathrm{E}_{\mathrm{RE}} \cdot \mathrm{N} \cdot \mathrm{P}_{\mathrm{e}}}{\varepsilon}=\rho \cdot \mathrm{D} \cdot \mathrm{Cp} \cdot \Delta \mathrm{T} \cdot \mathrm{N} \cdot \frac{\mathrm{P}_{\mathrm{e}}}{\varepsilon}(100-\% \mathrm{R}) \cdot 10^{-2}
\end{aligned}
$$

D'où le coût total des dépenses énergétiques annuelles en fonction du pourcentage de récupération d'énergie.

$$
\mathrm{C}_{\mathrm{N}}=\rho \cdot \mathrm{D} \cdot \mathrm{Cp} \cdot \Delta \mathrm{T} \cdot \mathrm{N} \cdot 10^{-2}(100-\% \mathrm{R})\left(\mathrm{Pt}+\frac{\mathrm{P}_{\mathrm{e}}}{\varepsilon}\right)
$$

Cette formule comporte tous les paramètres influençant le coût de l'énergie dépensée. Ce dernier sera proportionnel au débit de lait, à la durée du travail et à l'écart de température subi par le lait. 


\section{2b COÂT DE L'INVESTISSEMENT SUPPLÉMENTAIRE}

Le pasteurisateur schématisé à la figure 1 comporte un bâti, deux plateaux intermédiaires et les plaques constituant les trois zones d'échange.

Dans la mesure où notre méthode ne concerne que le coût des investissements destinés à récupérer plus de 80 p. 100 d'énergie, le coût du bâti et des plateaux intermédiaires est négligé. Seul le prix des plaques supplémentaires va intervenir.

La fonction établissant le coût annuel de l'investissement supplémentaire $\left(\mathrm{C}_{\mathrm{I}}\right)$ sera donc de la forme :

$$
\mathrm{C}_{\mathrm{I}}=\frac{\mathrm{S} . \mathrm{Pm}}{\mathrm{A}}
$$

La durée d'amortissement (A) permet de se ramener directement au coût annuel.

L'évolution de la surface nécessaire (S) en fonction du pourcentage de récupération ( $\% \mathrm{R})$ est liée au programme thermique mis en œuvre et aux caractéristiques de l'échangeur. Elle est obtenue par un calcul informatique. La croissance de $S$ est très rapide lorsque le pourcentage de récupération dépasse 90 p. 100 ; il en est évidemment de même pour le coût de l'investissement supplémentaire qui lui est directement proportionnel.

En première approximation, il est possible de considérer que le coût de cet investissement est également proportionnel au débit de lait traité. Ceci est illustré par la figure 2. Dans ces conditions, un

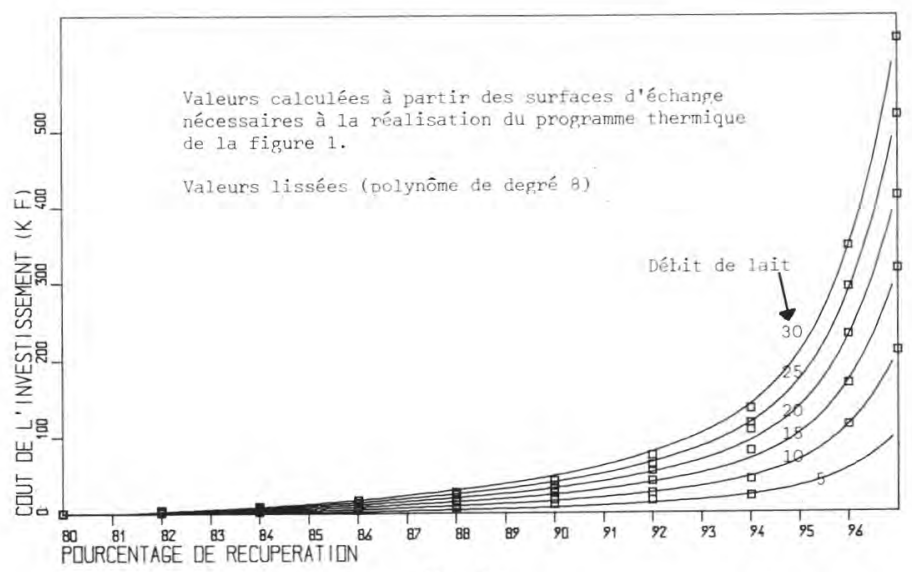

fig. 2

Evolution du coût de l'investissement en fonction du pourcentage de récupération pour différents débits (exprimés en $\mathrm{m}^{3} / \mathrm{h}$ ). 
polynome de degré 8 permet une description mathématique correcte et suffisamment précise de cette évolution :

$$
\mathrm{C}_{\mathrm{t}}=\text { D. }\left(\mathrm{b}_{0}+\mathrm{b}_{1} \cdot \mathrm{x}+\mathrm{b}_{2} \cdot \mathrm{x}^{2}+\ldots+\mathrm{b}_{8} \cdot \mathrm{x}^{8}\right)
$$

Pour les données numériques correspondant aux échangeurs Vicarb, la valeur des coefficients de régression est la suivante :

$$
\begin{aligned}
& b_{0}=1,1949 \\
& b_{1}=1,1538 \\
& b_{2}=0,9739
\end{aligned}
$$$$
\mathrm{b}_{3}=0,8468
$$$$
\mathrm{b}_{6}=0,0554
$$$$
\mathrm{b}_{4}=0,1955
$$$$
\mathrm{b}_{7}=0,4847
$$$$
\mathrm{b}_{5}=0,4877
$$$$
b_{8}=0,1869
$$

$$
\% \mathrm{R}-88,637
$$

avec $\mathrm{x}: \frac{\mathrm{R}}{5,504}$

Notre méthode qui prend pour référence du coût d'investissement, le prix du pasteurisateur dans sa configuration récupérant 80 p. 100 de l'énergie, reste applicable si le pourcentage de récupération, devenu très important (plus de 95 p. 100), exige l'utilisation d'un modèle de taille supérieure.

\section{Utilisation pratique}

La méthode proposée peut être utilisée pour définir le pourcentage de récupération optimum d'énergie d'une installation de pasteurisation à créer ou déjà en service.

Le coût des énergies dépensées est calculé en appliquant la relation (I). Les grandeurs intervenant dans cette relation sont, soit fixées par les conditions de travail, soit considérées comme des paramètres susceptibles d'être fixés pour conduire à une optimisation plus globale de la dépense énergétique.

Le coût de l'investissement supplémentaire est plus difficile à évaluer dans la mesure où la collaboration du constructeur du pasteurisateur est nécessaire. A priori, tout constructeur fournira soit les coûts d'investissement correspondant aux différentes possibilités de fonctionnement envisagées soit une fonction semblable à la relation (II) avec ses limites d'utilisation.

Lorsque les coûts d'investissement et de dépenses énergétiques sont établis en fonction du pourcentage de récupération d'énergie, il suffit d'en faire la somme et de tracer l'évolution du total obtenu en fonction du pourcentage de récupération. L'observation du tracé permet de déterminer sans difficulté la valeur minimale du coût total et par suite le pourcentage optimum de récupération d'énergie qui lui correspond. L'intervention des constructeurs pourrait aller jusqu'au calcul systématique du pourcentage optimum de récupération d'énergie. Ce calcul compléterait celui de l'échangeur en n'exigeant de la part de l'utilisateur que quelques données supplémentaires. 


\section{ETUDE NUMERIQUE}

\section{Position du problème}

L'objet de ce paragraphe est, en utilisant la méthode présentée, de fournir à partir de données numériques englobant la majorité des situations industrielles, les principales tendances traduisant l'évolution du pourcentage optimum de récupération d'énergie en fonction des principaux paramètres susceptibles de l'influencer.

A cet effet, nous avons étudié, en utilisant comme fonction " coût total " la somme des relations (I) et (II), les paramètres suivants :

- débit de lait ; intervalle d'étude : 5 à $30 \mathrm{~m}^{3} / \mathrm{h}$;

- durée d'amortissement de l'investissement supplémentaire ; intervalle d'étude 0,5 à 8 ans ;

— durée journalière de travail ; intervalle d'étude : 8 à 24 h/jour soit avec l'hypothèse de 300 jours de travail par an, 2400 à $7200 \mathrm{~h} / \mathrm{an}$ (pour simplifier, le temps nécessaire au nettoyage et à la désinfection est compris dans la durée journalière de travail avec une dépense énergétique supposée égale à celle du traitement) ;

- écart de température subi par le lait ; intervalle d'étude 70 à $90^{\circ} \mathrm{C}$ soit avec l'hypothèse que le lait entre et sort du pasteurisateur à $5^{\circ} \mathrm{C}$, des températures de pasteurisation comprises entre 75 et $95^{\circ} \mathrm{C}$;

- augmentation du coût de l'énergie ; hypothèse : hausses de 10 p. 100,25 p. 100,50 p. 100 et 100 p. 100 .

Par ailleurs, nous avons apprécié l'effet de l'encrassement progressif du pasteurisateur sur le pourcentage optimum de récupération d'énergie.

\section{Evaluation du prix de la thermie et de la frigorie}

Cette double évaluation est délicate car chaque usine, chaque unité de production a un prix de l'énergie qui lui est propre... et les écarts sont parfois importants. Ceci s'explique par la multiplicité des installations existantes, par leurs conditions de réglage, d'entretien et de fonctionnement, parfois très différentes et souvent éloignées des conditions idéales. De plus, selon la consommation énergétique de l'usine et selon sa localisation, les tarifs de fourniture peuvent varier. Enfin, les modes de comptabilités changent d'une usine à l'autre, sans toujours avoir la précision souhaitable essentiellement parce que les bilans énergétiques sont établis globalement et non appareil par appareil.

En conséquence, les données que nous avons utilisées, bien qu'issues de sources différentes que nous avons recoupées, ne satis- 
feront pas tous les cas de figure. Elles suffisent pour dégager les tendances recherchées. Mais, en pratique chaque intéressé devra reprendre la méthode préconisée en utilisant les données énergétiques propres à son unité de production.

Pour la production de chaleur, nous avons considéré que la thermie produite par condensation de vapeur à une température de $90^{\circ} \mathrm{C}$ coûtait $12 \mathrm{c}$ soit près de $70 \mathrm{~F}$ pour $1 \mathrm{t}$ de vapeur.

Pour la production de froid, nous avons d'abord considéré qu'un groupe frigorifique consommant $1 \mathrm{Kw} . \mathrm{h}$ produisait environ 2000 frigories sous forme d'eau glacée à $2^{\circ} \mathrm{C}$. Pour chiffrer le coût des dépenses d'électricité, nous avons appliqué le tarif E.D.F. de février 1980 [9.]. Le coût moyen du kilowatt-heure (en $\mathrm{kg}$ de Francs) en fonction du nombre d'heures par jour (n) s'exprime par la relation :

$$
\mathrm{Pe}=10^{-3}\left(3,574 \cdot 10^{-1}-1,190 \cdot 10^{-2} \cdot \mathrm{n}+2,412 \cdot 10^{-4}+\mathrm{n}^{2}\right)
$$

Notre analyse n'est pas assez proche de la réalité industrielle pour intégrer les possibilités de production d'eau glacée en " heures creuses ».

\section{Résultats et discussions}

\subsection{Remarques GÉNÉRALES}

La figure 3 comporte différentes courbes d'évolution de la fonction «coût annuel total » tracées comme il est indiqué précédemment. Il apparaît que chaque courbe présente un minimum significatif. La détermination du pourcentage de récupération d'énergie

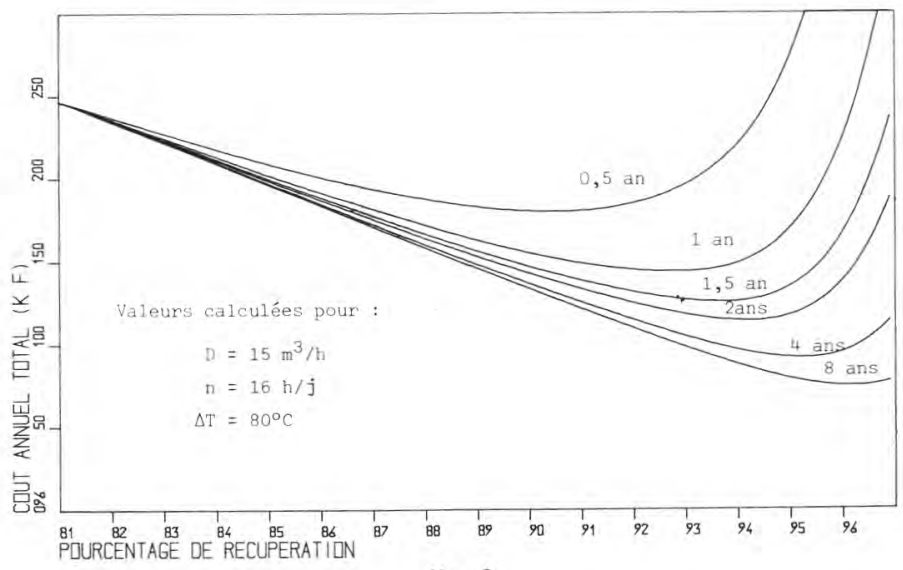

fig. 3

Exemple d'évolution du coût annuel total en fonction du pourcentage de récupération pour différentes durées d'amortissement. 


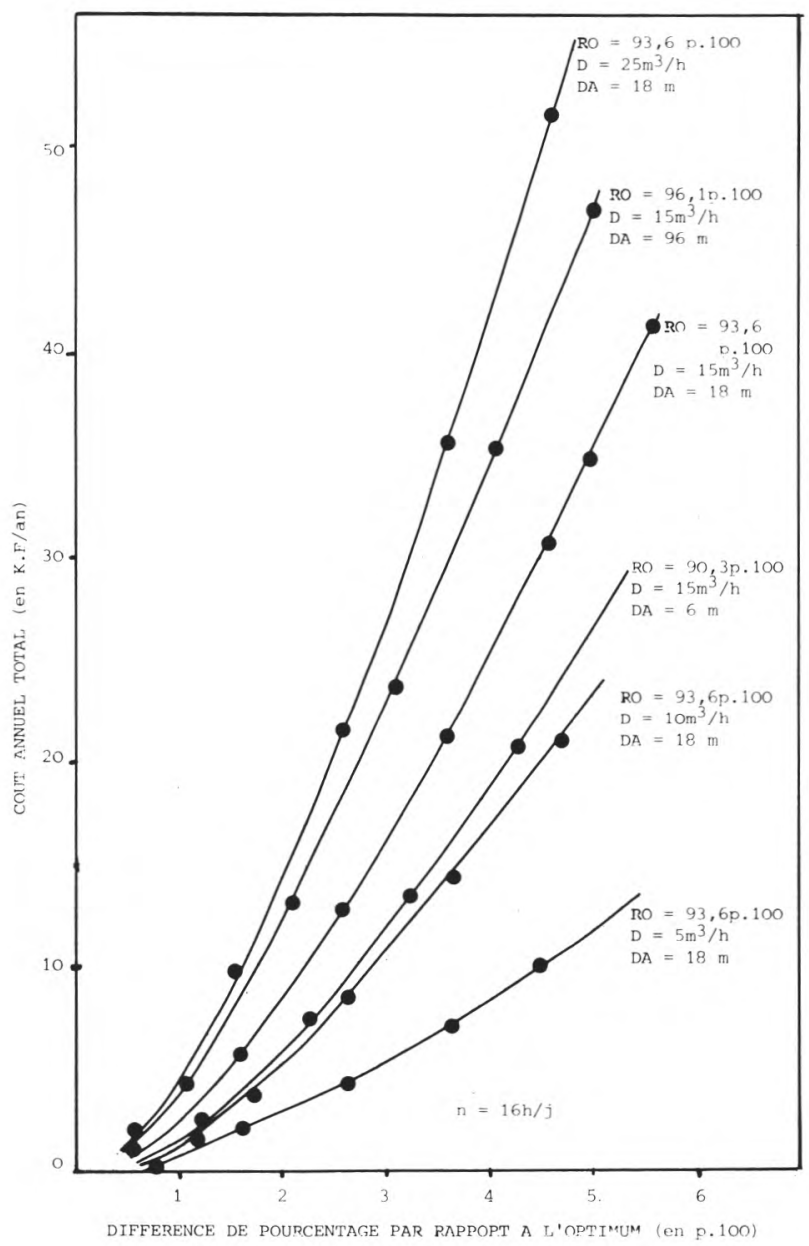

fig. 4

Evolution du coût annuel résultant d'une différence négative entre le pourcentage d'énergie récupérée et le pourcentage optimum.

optimum est donc un problème réel dont le résultat sera influencé par les principaux paramètres étudiés. Avant de les examiner successivement, deux remarques s'imposent :

- les valeurs de l'optimum obtenues varient, selon les conditions de travail, entre 90 p. 100 et 96 p. 100 de l'énergie totale mise en œuvre. Ces valeurs ne constituent pas une surprise mais confirment la nécessité de récupérations importantes ; 
- l'optimum est plus ou moins « pointu » selon la valeur des paramètres. Cela est traduit par le coût annuel résultant d'une différence existant entre le pourcentage d'énergie récupérée et le pourcentage optimum. La figure 4 quantifie l'évolution des valeurs négatives de cette différence dans les conditions de travail correspondant au tracé de la figure 3 . Le coût annuel croît avec la valeur absolue de l'optimum d'autant plus fortement que la différence est importante. Ainsi, d'après la figure 4, l'incidence financière annuelle d'une différence de 1 p. 100 sera inférieure à $5000 \mathrm{~F}$ mais pourra dépasser $50000 \mathrm{~F}$ pour une différence de 5 p. 100.

\subsection{INFLUENCE DES PRINCIPAUX PARAMÈTRES}

a) Définition de la valeur moyenne de chaque paramètre.

Pour montrer l'influence de chaque paramètre sur le pourcentage de récupération optimum, nous n'avons pas procédé à toutes les combinaisons possibles mais seulement étudié l'influence d'un paramètre, les autres étant fixés à une valeur moyenne. Il est ainsi possible de dégager des tendances générales. Nous avons respectivement choisi :

- un débit de $15 \mathrm{~m}^{3} / \mathrm{h}$;

- une durée d'amortissement de 18 mois ;

- une durée journalière de travail de $16 \mathrm{~h}$;

- un écart de température subi par le lait de $80^{\circ} \mathrm{C}$, soit une température de pasteurisation de $85^{\circ} \mathrm{C}$;

- le coût actuel de l'énergie.

Le pourcentage optimum de récupération est, dans ces conditions de 93,6 p. 100. C'est à partir de ce point qu'a été établie la figure 5 afin de représenter les variations de l'optimum en fonction des différents paramètres étudiés. Parallèlement, les principaux résultats sont discutés ci-dessous :

\section{b) Influence du débit de lait}

Compte tenu des approximations décrites précédemment, chacune des fonctions coût de l'énergie et coût de l'investissement supplémentaire est directement proportionnelle au débit de traitement. Il en résulte que le pourcentage de récupération optimum n'est pas influencé par le débit. Ce résultat est important. Il montre que le choix optimal ne dépend pas de la taille de l'unité de pasteurisation.

\section{c) Influence de la durée d'amortissement}

La figure 5 démontre l'importance considérable de la durée d'amortissement sur le pourcentage optimum de récupération d'énergie. Ce dernier est d'autant plus élevé que la durée d'amortissement est longue. Seules des durées d'amortissement de 6 mois conduiront, 


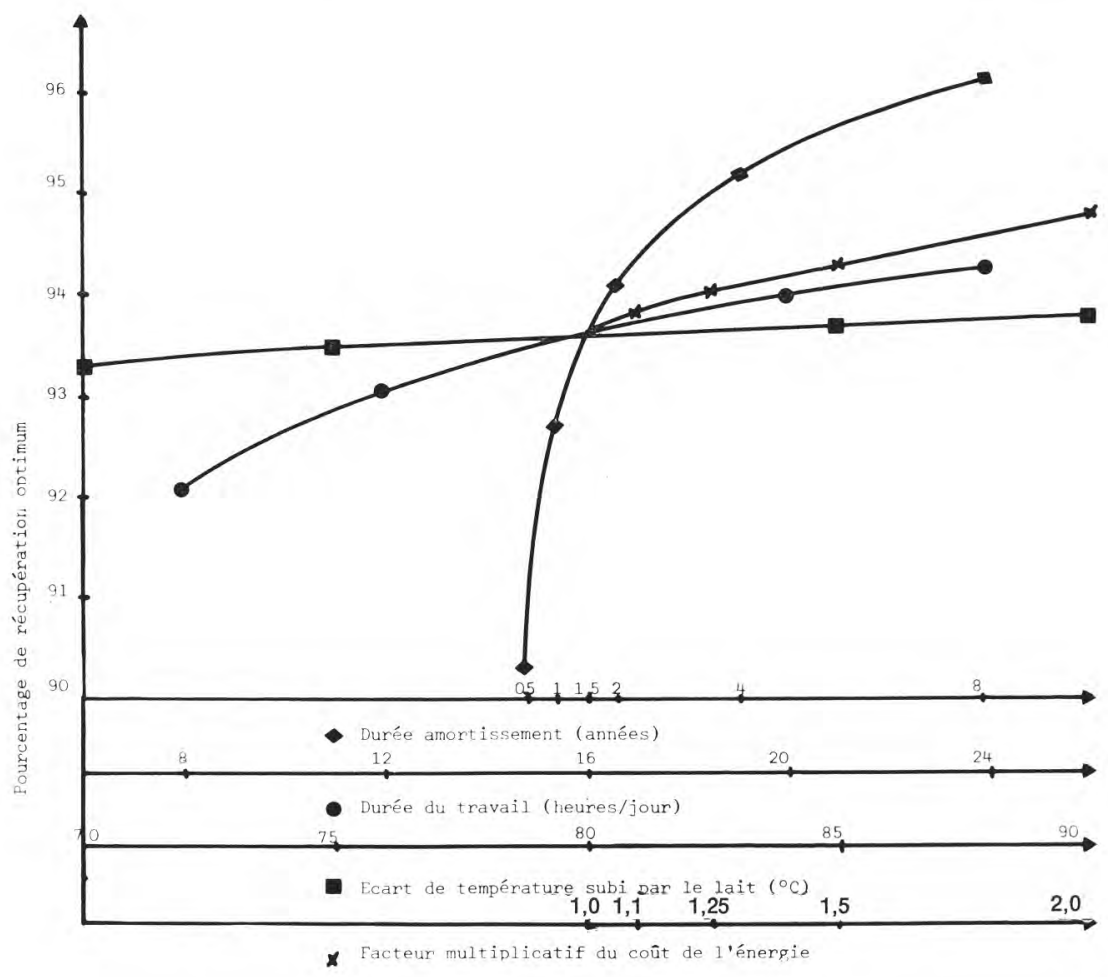

fig. 5

Influence des divers paramètres sur le pourcentage de récupération optimum.

pour de courtes durées de travail, à des pourcentages optimums inférieurs à 90 p. 100. Au contraire, de longues durées d'amortissement (4 et 8 années) correspondent à des pourcentages optimums supérieurs à 95 p.100. Les durées habituelles d'amortissement des investissements destinés à économiser l'énergie (18 à 24 mois) conduisent à des pourcentages de récupération compris entre 92 95 p. 100.

La durée d'amortissement de l'investissement supplémentaire est donc un paramètre fondamental pour la définition du pourcentage optimum de récupération d'énergie à mettre en œuvre. Dans la période actuelle où, outre le coût propre de l'énergie, il est indispensable de prendre en compte son incidence sur l'équilibre de notre balance commerciale, peut-être faut-il réviser les durées d'amor- 
tissement acceptables. Si l'on considérait qu'un équipement destiné à récupérer de l'énergie s'amortissait dans les mêmes conditions qu'un équipement productif (5 à 8 années), des pourcentages de récupération d'énergie supérieurs à 95 ou 96 p. 100 s'imposeraient dès à présent.

\section{d) Influence de la durée journalière de travail}

La figure 5 indique que l'optimum de récupération augmente avec la durée du travail. L'influence de ce paramètre n'est pas négligeable puisque l'optimum passe de $92,1 \mathrm{p} .100$ pour $8 \mathrm{~h} / \mathrm{j}$ à 94,3 p. 100 pour $24 \mathrm{~h} / \mathrm{j}$. Mais, il est délicat de totalement dissocier au niveau de ce paramètre le coût sans cesse croissant de la main-d'œuvre.

\section{e) Influence de la température de pasteurisation}

La température de pasteurisation affecte l'énergie mise en œuvre au sein du procédé et donc les coûts correspondants. Nos calculs ont montré que les investissements nécessaires à la récupération d'énergie sont relativement voisins pour chacun des pourcentages considérés entre 80 p. 100 et 97 p. 100 . Les écarts moyens sont de l'ordre de 5 à 10 p. 100 . En conséquence, nous avons considéré un coût d'investissement supplémentaire constant.

La figure 5 montre que l'optimum de récupération augmente de 93,3 p. 100 à 93,7 p. 100 quand la température de pasteurisation passe de $75^{\circ} \mathrm{C}$ à $95^{\circ} \mathrm{C}$. Ce paramètre n'est donc pas essentiel pour définir la récupération d'énergie optimale à mettre en œuvre.

\section{f) Effet du coût de l'énergie}

Le pourcentage de récupération d'énergie que nous déterminons est valable à l'instant du calcul. Nous n'avons pas inclus dans ce calcul une formule prenant en compte l'augmentation prévisible du coût de l'énergie pendant la durée d'amortissement car cette prévision est très délicate. En conséquence, nous avons étudié l'effet de hausses plus ou moins importantes du coût de l'énergie sur l'optimum de récupération.

La figure 5 indique que quand le prix de l'énergie double, le pourcentage optimum passe de 93,6 à 94,8 p. 100 .

En pratique, ce résultat peut conduire l'utilisateur à surévaluer légèrement son investissement pour tenir compte de la hausse inévitable de l'énergie durant la période d'amortissement qu'il a choisi.

\section{g) Effet de l'encrassement du pasteurisateur}

L'encrassement d'un pasteurisateur dans sa zone de chauffage ne modifie que les conditions du transfert de chaleur sans affecter la quantité d'énergie transférée.

Par contre, l'encrassement de la zone de récupération, sensible lorsque la température du lait dépasse $70^{\circ} \mathrm{C}[8]$, diminue le pour- 
centage d'énergie récupérée. Cela se traduit par un programme thermique légèrement différent : moins d'énergie étant récupérée, une compensation doit être apportée par le chauffage. Les conclusions de l'influence de la température de pasteurisation sur le pourcentage optimum de récupération s'appliquent donc partiellement à l'encrassement. Cela signifie que l'encrassement essentiellement pour des températures de pasteurisation égales ou supérieures à $85^{\circ} \mathrm{C}$, tend à augmenter le pourcentage de récupération optimum. Cependant, cette augmentation ne saurait excéder 0,1 p. 100 ou 0,2 p. 100 ce qui rend ce phénomène relativement peu important.

\section{CONCLUSIONS}

La méthode proposée pour déterminer le pourcentage de récupération d'énergie optimum sur un pasteurisateur de lait à plaques consiste :

$1^{\circ}$ A traiter le problème énergétique indépendamment des autres problèmes d'installation et de fonctionnement.

$2^{\circ}$ A considérer que l'investissement supplémentaire pour récupérer plus de 80 p. 100 de l'énergie doit être amorti par l'énergie récupérée et elle seule.

$3^{\circ}$ A déterminer pour chaque pourcentage de récupération d'énergie étudié entre 80 et 97 p. 100, une configuration du circuit de lait assurant une perte de charge constante. Cela permet de négliger les coûts d'investissement relatif au pompage (augmentation de la taille ; puissance électrique consommée).

Dès lors, la méthode peut s'appliquer à un pasteurisateur à installer ou en fonctionnement, même si ce dernier est amorti.

L'établissement de la fonction coût d'énergie dépensée résulte de l'analyse du procédé. L'évaluation correcte des prix de la thermie et de la frigorie, dans la mesure où chaque usine ou unité de production a un prix qui lui est propre, est indispensable.

L'établissement de la fonction coût de l'investissement supplémentaire exige la collaboration du constructeur du pasteurisateur.

La représentation graphique de la somme des deux fonctions coût permet de déterminer visuellement l'optimum recherché.

L'application numérique présentée englobe la majorité des situations industrielles et se base sur des coûts énergétiques significatifs à la date du $1^{\text {er }}$ juin 1980. Ainsi, nous avons pu analyser l'effet des principaux paramètres agissant sur le pourcentage de récupération d'énergie optimum. 
Les conclusions de l'étude sont :

- la démonstration graphique de la réalité d'un pourcentage optimum de récupération d'énergie ;

- le caractère relativement " pointu » de l'optimum qui s'accentue lorsque le pourcentage de récupération croît. Une différence de 5 p. 100 entre les pourcentages d'énergie optimum et réellement récupérée peut majorer le coût annuel d'exploitation d'environ $50000 \mathrm{~F}$;

- l'influence prépondérante de la durée d'amortissement considérée. Les durées habituelles de 1,5 à 2 années conduisent selon les conditions de fonctionnement à des pourcentages optimums généralement compris entre 92 et 95 p. 100 de récupération d'énergie. Seuls de très rares cas de figures justifient encore une récupération inférieure à 90 p. 100 . Si une évolution politico-économique conduisait les industriels à amortir les investissements destinés à économiser l'énergie de la même façon que ceux destinés à la production (durées d'amortissement de 5 à 8 ans), la récupération optimale d'énergie serait comprise entre 95 et 97 p. 100 ;

- l'augmentation du temps de travail bien que moins influente que la durée d'amortissement tend à augmenter le pourcentage optimum de récupération d'énergie, par contre le débit de traitement n'a pratiquement aucune influence ;

- la température de pasteurisation, reflet du programme thermique mis en œuvre, et l'encrassement progressif du pasteurisateur, reflet de ses conditions de fonctionnement, n'ont qu'un effet mineur sur l'optimum à considérer ; il n'augmentera que faiblement sous leur influence conjuguée ;

- l'augmentation inévitable du coût de l'énergie fera encore croître le pourcentage optimum de récupération. Néanmoins, la taille des pasteurisateurs à mettre en œuvre serait telle que des récupérations supérieures à 98 p. 100 de l'énergie semblent peu probables même à assez long terme. Au contraire, en considérant que la surface totale des plaques d'un pasteurisateur traitent $15 \mathrm{~m}^{3} / \mathrm{h}$ de lait augmente de $83 \mathrm{~m}^{2}$ à $232 \mathrm{~m}^{2}$ quand le pourcentage de récupération d'énergie est porté de 90 à 95 p. 100, il faut s'interroger sur les risques de dégradation du lait. Dégradations rendues possibles par de longs temps de séjour dans l'appareil et par d'éventuels phénomènes de recontamination par développement de germes sur les surfaces de refroidissement $[4,6]$.

Concluons en remarquant que la pasteurisation est l'un des procédés de l'agro-alimentaire parmi les plus économiques en énergie ; cela est d'autant plus vrai que l'optimisation de la récupération est réalisée. Cette étude devrait sensibiliser les utilisateurs aux possibilités d'optimisation et les amener à reconsidérer périodiquement le fonctionnement énergétique de leurs pasteurisateurs. 


\section{Symboles et unités}

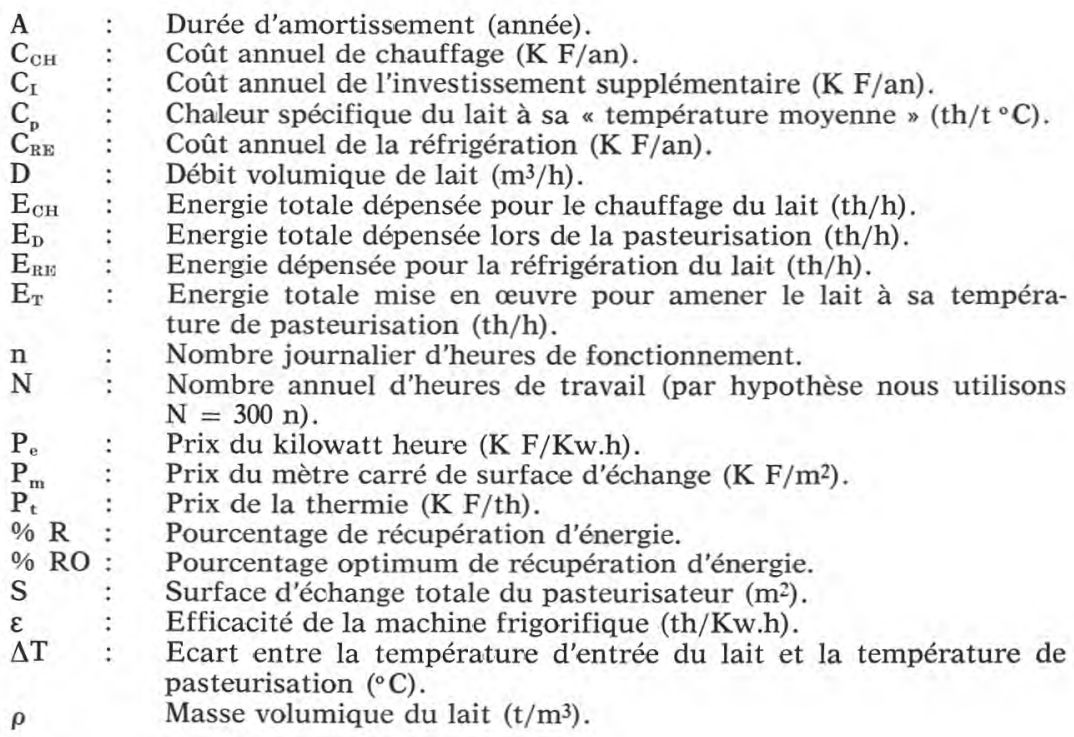

\section{Résu mé}

Les auteurs proposent une méthode simplifiée pour le calcul de la récupération optimum d'énergie sur un pasteurisateur de lait à plaques. Cette méthode suppose de traiter séparément le problème énergétique en considérant que l'investissement permettant des récupérations supérieures à 80 p. 100 est amorti par l'économie réalisée.

Une étude numérique montre le rôle essentiel de la durée d'amortissement considérée. Les autres paramètres étudiés (débit de lait, durée journalière de travail, programme thermique mis en œuvre, degré d'encrassement, coût de l'énergie) ont un effet plus faible, parfois négligeable.

\section{S u $\mathrm{m} \mathbf{m}$ a r y}

\section{SIMPLIFIED METHOD TO CALCULATE THE OPTIMUM ENERGY RECOVERY ON A PLATE TYPE MILK PASTEURIZER}

A simplified method to calculate the optimum energy recovery on a plate type milk pasteurizer is proposed: The investment necessary for an energy saving up to $80 \mathrm{p} .100$ of the total energy required is wroten off only by the reduction of the energy consumption. 
A numeric study shows that the writing off duration is the most significant parameter. Others parameters (temperature, daily working time, costs of energy, fouling) have smaller effects.

Reçu pour publication en novembre 1980.

\section{Bibliographie}

[1] Clayton (D. G.) (1974). - New Concepts for Heat Exchanger Performance. Wärme und Stoffübertrangung, 7 (2), 107.

[2] CORRIEU (G.) (1980). - Quelques considérations sur les échangeurs de chaleur à plaques. La Technique Laitière, 941, 29-39 ; 942, 11-13.

[3] Derkenne (J.) (1980). - Energie : bilan d'un an d'expertise dans les usines laitières. La Technique Laitière, 944, 37-39.

[4] Driessen (F. M.) \& Bouman (S.) (1979). - Groei van thermoresistente streptokokken in kaasmelkpasteurs. 2. Proefnemingen met een modelpasteur. Overdruk uit Zuivelzicht, 48, 71 jaargang, 1062-1064.

[5] FraAs (A. P.) and OzisiK (M. N.) (1965). - Heat Exchange Design. John Wiley and Sons, New York.

[6] Hup (G.), Bangma (A.), Stadhouders (J.) \& Bouman (S.) (1979). - Groei van thermoresistente streptokokken in kaasmelkpasteurs. 1. Enkele waarnemingen in kaasbedrijven. Overdruk uit Zuivelzicht, $46,71^{\mathrm{e}}$ jaargang, 1014-1016.

[7] Jenssen (S. K.) (1969). - Heat Exchanger Optmization. Chem. Eng. Prog., $65(7), 59$.

[8] Lalande (M.), Corrieu (G.), Tissier (J. P.) et Ferret (R.) (1979). - Etude du comportement d'un échangeur à plaques Vicarb utilisé pour la pasteurisation du lait. Le Lait, 581-582, 13-33.

[9] Le Goff (R.), Almin (Y.), Deschamps (G.) et Laroche (G.) (1980). - La pompe à chaleur et ses applications industrielles. Etude E.D.F. HE 142 T 403.

[10] TABOREK (J. J.) (1969). - Organisation of Heat Exchanger Programs on Digital Computers. C.E.P. Symp. Series 54 (20), 207.

[11] Thompson (D. R.) (1977). - Energy Analysis in Heating and Cooling Processes. Food Technol., 31, 3, 51-56.

[12] Warshney (N. N.), Narkhede (D. D.), OJHa (T. P.) (1979). - Modified HighTemperature-Short-Time Cream Pasteurizer for Low Energy Consumption. Food Sc. Technol., 16, 54-57.

Cet article a fait l'objet d'une communication au Symposium International "Energie et Industries Agricoles et Alimentaires ", Madrid, 6-7-8 octobre 1980. 